\title{
CULTURA MAPUCHE: ENTRE LA PERTENENCIA ÉTNICA Y LA INTEGRACIÓN NACIONAL
}

\author{
Ignacio Irarrázaval \\ y María de los Ángeles Morandé
}

\begin{abstract}
A la luz de los resultados de la encuesta mapuche CEP (2006), en este trabajo se estudia el grado de intensidad con que diferentes grupos al interior del mundo mapuche se identifican con su cultura originaria, versus su integración a la cultura nacional. Para ello se analizan las percepciones y actitudes de la población mapuche respecto a la práctica y mantención de su cultura, así como sus expectativas de integración a la cultura nacional. El estudio muestra que en el "sentir mapuche” coexistirían una pertenencia más bien simbólica a la cultura ancestral y anhelos reales de integración a la cultura nacional.
\end{abstract}

Ignacio Irarrázaval. Ph. D. Política Social London School of Economics. Director de Asuntos Públicos Pontificia Universidad Católica de Chile y profesor de Políticas Públicas de la misma universidad.

María de los Ángeles Morandé. Socióloga Pontificia Universidad Católica de Chile. Estudiante de Magíster en Desarrollo Urbano de la misma universidad.

N. del E.: Sobre los resultados de la encuesta mapuche realizada por el CEP (2006), véanse también en esta edición los trabajos de Fernando Zúñiga, Eduardo Valenzuela, Aldo Mascareño, Rolf Foerster y Sonia Montecino, Juan M. Ossio, así como el comentario de Jorge Larraín al artículo de Aldo Mascareño y la presentación de Carolina Segovia y Lucas Sierra. 


\section{Introducción}

L públicas nacionales hace necesario detenerse a reflexionar acerca de las percepciones y expectativas reales de quienes dicen pertenecer a esta cultura originaria, para contar así con mejores elementos de análisis sobre sus necesidades y sentir.

El presente trabajo forma parte de una serie de otros estudios cuyo objetivo es conocer y comprender mejor el mundo mapuche a partir del análisis de los resultados de la encuesta realizada por el Centro de Estudios Públicos en el primer semestre del 2006, que abarcó de manera representativa a la población mapuche del país.

A modo de ficha técnica, la encuesta recopiló información a través de cuestionarios aplicados presencialmente a 1.487 casos de personas mapuche* distribuidas en las regiones de mayor concentración de la etnia en Chile, de acuerdo a información censal. Se consideró además un grupo de control, para una muestra de igual tamaño, de personas no mapuche distribuidas en las mismas zonas. En este sentido, se trata de un grupo no mapuche que sólo se diferencia de la muestra mapuche por no pertenecer a un pueblo originario. Ambas muestras probabilísticas e independientes consideraron un error muestral de 2,7 puntos porcentuales con un 95\% de confianza.

Si bien la encuesta abarcó una amplia variedad de temas, el análisis aquí presentado sólo considera algunos de ellos, intentando rescatar información principalmente atingente a las percepciones y actitudes respecto a la pertenencia a la cultura mapuche y a las expectativas de integración de la población mapuche.

Cabe hacer referencia a ciertas limitaciones presentes en este estudio a la hora de analizar la intensidad de la pertenencia a la cultura mapuche, pues hubo que ceñirse a las preguntas ya aplicadas en la encuesta para realizar el análisis y los cruces estadísticos correspondientes. Sin embargo, el trabajo propuesto pretende reflejar el sentido e intensidad de pertenencia del pueblo mapuche a su cultura y la conformación de una identidad étnica en base a las expectativas de integración a la identidad nacional.

* Los autores de este artículo escriben "personas mapuche" y no "personas mapuches" en atención al componente "che” (gente) del vocablo "mapuche”. (N. del E.) 


\section{Marco conceptual: Pertenencia a la cultura mapuche}

Los pueblos originarios de nuestro país son reconocidos por el Estado chileno como "los descendientes de las agrupaciones humanas que existen en el territorio nacional desde tiempos precolombinos, que conservan manifestaciones étnicas y culturales propias, siendo para ellos la tierra el fundamento principal de su existencia y cultura"1. Estos pueblos representan de acuerdo al Censo 2002, el 4,6\% de la población de nuestro país, y la etnia mapuche es la más numerosa de la población indígena, pues alcanza un total de 602.677 personas, que corresponden al $87 \%$ del total de población indígena chilena.

Los mapuche se concentran en las regiones VIII, IX, X y Región Metropolitana, distribuyéndose en un 63\% en zonas urbanas. Esto último responde principalmente a los movimientos migratorios en que se han visto envueltos los mapuche, quienes después de constituirse por años como una cultura más bien rural, han estado trasladándose en el último medio siglo hacia la ciudad en busca de mayores oportunidades laborales y económicas.

De acuerdo al Censo 2002, se definen como mapuche aquellas personas que se autoidentificaron como tales en la pregunta "¿Pertenece usted a alguno de los siguientes pueblos originarios o indígenas?”. En el caso de la encuesta aquí analizada se empleó la misma pregunta y se aplicó el cuestionario a aquellas personas mayores de 18 años que se declararon pertenecientes al pueblo mapuche y que habitan las regiones de mayor concentración de población mapuche antes señaladas.

Ahora bien, conviene detenerse un momento en la pertenencia autodeclarada a la cultura mapuche, pues numerosas han sido las discusiones que se han planteado en nuestro país en torno a la pregunta censal de identificación de la población originaria, empleándose en el último censo una pregunta dirigida particularmente a la pertenencia a un pueblo originario para cuantificar a la población de origen indígena. Esto ha traído a la mesa temas tales como si la pertenencia a la cultura mapuche se relaciona directamente con la práctica de sus costumbres y con la configuración de una identidad étnica o si solamente tiene que ver con una adhesión personal y voluntaria a una cultura ancestral.

Hay autores cercanos al mundo indígena que se han inclinado hacia esta última postura, afirmando que los indígenas pueden mantener su identidad al margen de participar en la que identifican como su cultura de origen (Eliseo Cañulef, 1998). En este sentido, el indígena se autoidentificaría como

\footnotetext{
${ }^{1}$ Ley Indígena No 19.253, 1993, artículo 1.
} 
tal aunque se sienta miembro de una cultura mayor que no es indígena. De esta manera, se produce entre los indígenas, y especialmente entre los mapuche, una especie de ambivalencia cultural, pues en función de su permeabilidad a influencias externas (al igual que otras culturas) han debido acomodar sus estilos de vida y adaptarse a la cultura dominante en una tensión permanente con la mantención de su propia cultura y tradiciones.

$\mathrm{Al}$ respecto se puede afirmar que hoy en día el pueblo mapuche no tiene una identidad única, pues combina elementos, comparte estilos de vida y prácticas sociales con el resto de los ciudadanos chilenos, con los campesinos, obreros, o las trabajadoras de servicio doméstico, lo que los hace formar parte simultáneamente de una identidad nacional, además de la identidad étnica que les es propia. En este sentido, la identidad mapuche se va configurando en base a la experiencia individual y colectiva de acuerdo con los distintos roles y posiciones sociales que adquieren en su entorno.

Sin embargo, si bien la identidad cultural mapuche convive en cada sujeto con otras identidades sociales, su identidad étnica se mantiene viva entre los mapuche en distintas intensidades y se manifiesta en el reconocimiento de ciertos elementos constituyentes de su cultura. En esta dirección, el estudio "Línea Base en Comunidades Indígenas del Programa Orígenes” (Universidad de Chile, 2004) logró identificar algunos de estos elementos en un trabajo en terreno con los promotores del programa (todos de origen mapuche). Otros estudios también han hecho alusión a estos elementos como constituyentes de una etnia (F. Zúñiga, 2006; MINEDUC, 2005), entre los cuales se encuentran los siguientes que se describen a continuación.

En primer lugar el territorio (tuwun), que tal como reconoce la ley indígena es uno de los fundamentos principales de las culturas indígenas. Para los mapuche el sentido de territorialidad o de pertenencia a la tierra es, en un sentido amplio, un sentimiento de pertenencia a un entorno natural que los provee de recursos, aun viviendo lejos de ella. De esta manera, existe en los mapuche un vínculo histórico con la tierra, los recursos naturales y su entorno, que aunque no se traduzca necesariamente en su ocupación física, representa un apego simbólico al territorio ancestral, lugar donde descanan las raíces de su cultura.

En segundo lugar, la lengua, en tanto idioma propio y original de una etnia, es reconocida como uno de los aspectos más relevantes de la cultura mapuche por su papel cohesionante y por favorecer la continuidad cultural, basada principalmente en una tradición oral.

Otro elemento constituyente de la cultura mapuche dice relación con su cosmovisión o percepción general del mundo y de los principios que lo 
rigen, contenidos en la sabiduría mapuche (kimün) y expresadas en un sistema de creencias, en la religiosidad, en los agentes culturales, en su patrimonio cultural y lugares sagrados, que en conjunto determinan un creer y sentir común de su cultura.

Un cuarto elemento reconocido por los mapuche como constituyente de su identidad es su historia común, en tanto los recuerdos compartidos de las formas de vida, costumbres, tradiciones y rasgos físicos de sus antepasados se actualizan en su identidad como pueblo.

Por último, el linaje (kupan) es otro de los elementos integrales de esta cultura, en tanto que la sangre marca el sentido de descendencia de un ancestro mapuche que se representa en el recorrido histórico de los apellidos en el tronco familiar y apela a la idea de un origen común en el tiempo y en el espacio que otorga a la etnia un sentido de parentesco.

En definitiva, en el sentir mapuche aparecen recurrentemente estos elementos, los cuales en mayor o menor intensidad son una referencia simbólica a la cultura ancestral, que permanecen vivos en el imaginario mapuche.

\section{Metodología de análisis}

A partir de los conceptos revisados se pretende analizar las distintas intensidades con que los mapuche viven o perciben la pertenencia a su cultura y de qué manera esta identidad étnica convive actualmente con otras identidades sociales o con sus anhelos de integración a la identidad nacional. Desde esta perspectiva, y a partir de las preguntas de la encuesta y de los datos recabados, se construyó un índice para medir la intensidad de la pertenencia a la cultura mapuche en la muestra, intentando operacionalizar de la mejor manera posible los cinco elementos identificados como constituyentes de dicha cultura.

En primer lugar, se incorporó la práctica de ritos y ceremonias mapuche, en tanto manifestación de la adherencia a una cosmovisión o sistema de creencias ligadas a la pertenencia a la tierra y a un pasado en común. Se incluyó como variable la frecuencia de participación en costumbres como el ngillatun, wetripantu, machitún, visitas a la machi, visitas al longko ${ }^{2}$, en un subíndice de práctica de costumbres que consideró las categorías de: regularmente, esporádicamente y nunca.

${ }^{2}$ Ngillatun es una ceremonia de rogativa a espíritus ancestrales para una buena cosecha u otro beneficio de la comunidad; wetripantu es la celebración del año nuevo mapuche. El machitún es el ritual de sanación de enfermos. Machi es una autoridad de la medicina tradicional y conocedora de los secretos del mundo mapuche. El Longko es la máxima autoridad o cabeza dentro de una comunidad. 
En segundo lugar, se incorporó al índice el uso de la lengua como uno de los rasgos más relevantes en la conservación de una cultura. Se incluyeron en el índice, de acuerdo al conocimiento y uso de la lengua mapuche, tres grupos: aquellos que hablan la lengua, aquellos que la entienden pero no la hablan, y aquellos que no la hablan ni la entienden.

Por último, se consideró el reconocimiento de un linaje, es decir la identificación de presencia de sangre mapuche en sí mismos. Se consideró en el índice a aquellos que reconocen tener: mucha sangre mapuche, algo de sangre mapuche o nada de sangre mapuche.

De esta manera, se construyó un índice sumatorio ${ }^{3}$ de intensidad de la pertenencia a la cultura mapuche, el cual permitió clasificar a los encuestados en tres grupos: mapuche de alta intensidad de pertenencia, mapuche de media intensidad de pertenencia, y mapuche de baja intensidad de pertenencia, cuya distribución aparece en la Tabla $\mathrm{N}^{\mathrm{o}} 1 .^{4}$

La Tabla $N^{\circ} 2$ muestra el comportamiento de los distintos grupos en la muestra. Se puede observar que existe un grupo de alta intensidad (el $20 \%$ de los casos) que practica con mayor frecuencia las costumbres mapuche, la mayoría de ellos habla la lengua mapuche y reconocen tener mucha sangre mapuche.

Por su parte, en el grupo de intensidad media, que representa casi un tercio de la muestra, la mayoría practica esporádicamente las costumbres y casi un 30\% no lo hace nunca. Existe un conocimiento y uso menor de la lengua mapuche y, de hecho, un gran porcentaje no la habla ni la entiende, aunque la mayoría afirma tener mucha sangre mapuche.

TABLA No 1 :

GRUPOS DE INTENSIDAD DE LA PERTENENCIA A LA CULTURA MAPUCHE

Grupos de intensidad de pertenencia

Porcentaje

Alta intensidad

$19,6 \%$

Media intensidad

$31,7 \%$

Baja intensidad

$48,8 \%$

Total

$100 \%$

${ }^{3}$ Para la construcción del índice se asignó un valor de 1 a 3 a cada categoría de las variables, de acuerdo a la menor o mayor intensidad o práctica de las mismas, las cuales al sumarse en el índice permitieron clasificar los casos en los tres grupos de intensidad de la pertenencia.

${ }^{4}$ Todas las tablas y gráficos de este documento corresponden a datos de la encuesta mapuche realizada por el CEP (2006). 
TABLA No 2: $\quad$ PORCENTAJE DE PRÁCTICA DE COSTUMBRES, USO DE LA LENGUA Y RECONOCIMIENTO DE SANGRE MAPUCHE POR GRUPOS DE INTENSIDAD DE PERTENENCIA

\begin{tabular}{|c|c|c|c|c|}
\hline \multicolumn{2}{|c|}{$\begin{array}{l}\text { Variables/Grupos de } \\
\text { intensidad de pertenencia }\end{array}$} & $\begin{array}{c}\text { Alta } \\
\text { intensidad } \\
45 \%\end{array}$ & $\begin{array}{c}\text { Media } \\
\text { intensidad } \\
4 \%\end{array}$ & $\begin{array}{c}\text { Baja } \\
\text { intensidad } \\
\\
\text { - }\end{array}$ \\
\hline $\begin{array}{l}\text { Práctica } \\
\text { costumbres } \\
\text { mapuche }\end{array}$ & $\begin{array}{l}\text { Regularmente } \\
\text { Esporádicamente } \\
\text { Nunca }\end{array}$ & $\begin{array}{r}45 \% \\
55 \% \\
-\end{array}$ & $\begin{array}{r}4 \% \\
68 \% \\
28 \%\end{array}$ & $\begin{array}{r}- \\
12 \% \\
88 \%\end{array}$ \\
\hline & Total & $100 \%$ & $100 \%$ & $100 \%$ \\
\hline $\begin{array}{c}\text { Uso de la } \\
\text { lengua mapuche }\end{array}$ & $\begin{array}{c}\text { Habla } \\
\text { No habla, entiende } \\
\text { No habla ni entiende }\end{array}$ & $\begin{array}{r}91 \% \\
9 \% \\
-\end{array}$ & $\begin{array}{l}36 \% \\
45 \% \\
19 \%\end{array}$ & $\begin{array}{r}- \\
7 \% \\
93 \%\end{array}$ \\
\hline & Total & $100 \%$ & $100 \%$ & $100 \%$ \\
\hline $\begin{array}{l}\text { Reconocimiento } \\
\text { de sangre } \\
\text { mapuche }\end{array}$ & $\begin{array}{l}\text { Mucha sangre mapuche } \\
\text { Algo de sangre mapuche } \\
\text { Nada de sangre mapuche }\end{array}$ & $\begin{array}{r}98 \% \\
2 \% \\
-\end{array}$ & $\begin{array}{r}88 \% \\
12 \% \\
-\end{array}$ & $\begin{array}{r}33 \% \\
64 \% \\
3 \%\end{array}$ \\
\hline & Total & $100 \%$ & $100 \%$ & $100 \%$ \\
\hline
\end{tabular}

En contraste, predomina un grupo de baja intensidad de pertenencia que cubre prácticamente la mitad de la muestra y que si bien se autorreconoce como mapuche, en su mayoría no practica las costumbres mapuche, no habla ni entiende la lengua y sólo un tercio de ellos afirma tener mucha sangre mapuche.

Es necesario tener en consideración que no todos los mapuche viven y sienten su cultura de la misma manera. Si bien existe un grupo minoritario que practica la cultura con mayor intensidad, existe también un grupo que se siente igualmente perteneciente a esa cultura pero participa con otra intensidad de sus costumbres y creencias, concibiéndose a la vez como parte de una cultura nacional mayor. Esta coexistencia de identidades entre los mapuche es lo que se tratará de estudiar a lo largo de este trabajo a partir de las propias apreciaciones y aspiraciones de los mismos mapuche.

\section{Caracterización socioeconómica de los grupos de intensidad de pertenencia}

Antes de entrar en el detalle del análisis, conviene conocer un poco los grupos definidos de acuerdo a la intensidad de pertenencia. En primer 
TABLA N ${ }^{\circ}$ 3: $\quad$ DISTRIBUCIÓN DE LOS GRUPOS EN ZONA URBANA O RURAL

\begin{tabular}{lccc}
\hline Grupos de intensidad de pertenencia & Zona urbana & Zona rural & Total \\
\hline Alta intensidad & $28 \%$ & $72 \%$ & $100 \%$ \\
Media intensidad & $55 \%$ & $45 \%$ & $100 \%$ \\
Baja intensidad & $78 \%$ & $22 \%$ & $100 \%$ \\
Total & $61 \%$ & $39 \%$ & $100 \%$ \\
\hline
\end{tabular}

lugar, como se observa en la Tabla $\mathrm{N}^{\mathrm{o}} 3$, un alto porcentaje de los mapuche de los distintos grupos vive en zonas urbanas (61\%), como lo indican los resultados del Censo 2002 a nivel nacional. Sin embargo, esta situación varía al interior de cada de uno de los grupos, disminuyendo la proporción de mapuche urbanos a medida que aumenta la intensidad de la pertenencia. De esta manera, observamos que el grupo de alta intensidad de pertenencia se localiza en su mayoría en zonas rurales, los del grupo de intensidad media se distribuyen más parejos entre ambas zonas, y se revierte la situación en los mapuche de baja intensidad, quienes en su mayoría viven en áreas urbanas.

La ruralidad predominante en el grupo de alta pertenencia se relaciona con que un alto porcentaje de ellos (95\%) vive o tiene derechos sobre alguna comunidad mapuche, situación que en el caso de los mapuche de intensidad media de pertenencia alcanza sólo un 47\% y en los de baja intensidad un $15 \%$.

En cuanto a la situación socioeconómica de estos grupos, de acuerdo al Gráfico $\mathrm{N}^{0} 1$, se puede afirmar que se trata de un conjunto que se encuentra más bien en los últimos escalones de clasificación, pues en su mayoría pertenece a estratos bajos y en menor proporción a estratos medios, y no está representado el estrato alto en la muestra. El grupo de alta intensidad de pertenencia a la cultura mapuche coincide con ser el grupo más pobre, pues concentra el 88\% de su población en estos estratos. En relación con lo anterior, se trata de hogares que viven en condiciones bastante precarias. En el caso de los hogares mapuche de alta pertenencia, esto se refleja en la elevada proporción de viviendas que no cuentan con servicios básicos, como agua potable (57\%), sistemas de alcantarillado (72\%) o baño al interior de la vivienda (68\%), lo cual coincide con la mayor cantidad de hogares de este grupo que viven en zonas rurales.

La situación de pobreza de la población indígena a nivel nacional puede ser corroborada con datos de la encuesta CASEN 2003, en donde se constató que un 28,7\% de la población indígena en Chile vive bajo la línea 
GRÁFICO No ${ }^{\circ}$ : $\quad$ NIVEL SOCIOECONÓMICO POR GRUPOS DE INTENSIDAD DE PERTENENCIA CULTURAL

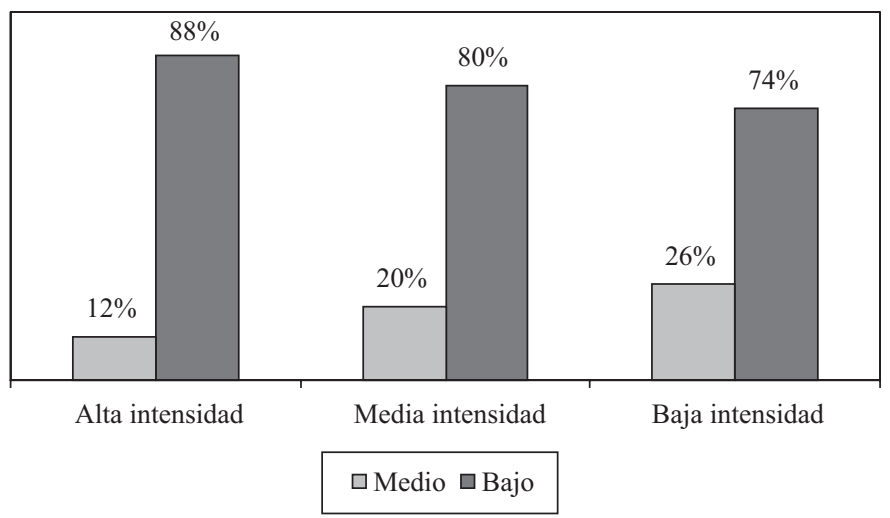

de pobreza. Asimismo, dicha encuesta demuestra que en zonas rurales estos niveles de pobreza son mayores, pues un 32\% de la población indígena es pobre o indigente en comparación con el 27\% que sufre esta condición en zonas urbanas. En cuanto al nivel de ingresos de esta población, dicho instrumento verifica que el 63\% de los indígenas se concentran en los dos quintiles más bajos de ingresos, correspondiendo principalmente a los grupos asentados en zonas rurales.

La pobreza en que vive en general la población mapuche en nuestro país se ha asociado generalmente con la naturaleza más bien de subsistencia de su economía. Sin embargo, su situación de pobreza se puede relacionar también con sus bajos niveles de escolaridad y calificación que les dificultan acceder a trabajos de mejor calidad. En este sentido, se puede ver en la muestra de estudio el bajo nivel de educación de esta población. Como ilustra la Tabla $\mathrm{N}^{\circ} 4$, se observa que el grupo de alta intensidad de pertenencia presenta menos años de estudio que los otros grupos. A este resultado se suma la alta tasa de analfabetismo, que en este grupo alcanza un $17 \%$, a diferencia de los grupos de media y baja intensidad de pertenencia, que presentan tasas de $7 \%$ y $5 \%$ respectivamente.

A nivel nacional según datos CASEN 2003, la tasa de analfabetismo en la población indígena alcanza el 8,4\%, predominando esta situación en zonas rurales con un 16\% de la población indígena analfabeta, tratándose especialmente de grupos etarios mayores de 60 años. Asimismo, los niveles educacionales son bastante bajos, pues un 9\% de la población indígena nacional no tiene educación formal y un 38\% de ellos tiene educación básica incompleta. 


\begin{tabular}{lccc}
\hline Años de estudio & $\begin{array}{c}\text { Alta } \\
\text { intensidad }\end{array}$ & $\begin{array}{c}\text { Media } \\
\text { intensidad }\end{array}$ & $\begin{array}{c}\text { Baja } \\
\text { intensidad }\end{array}$ \\
\hline $0-3$ años & $32 \%$ & $14 \%$ & $13 \%$ \\
$4-8$ años & $48 \%$ & $42 \%$ & $35 \%$ \\
$9-12$ años & $14 \%$ & $32 \%$ & $39 \%$ \\
Toños o más & $6 \%$ & $12 \%$ & $13 \%$ \\
& $100 \%$ & $100 \%$ & $100 \%$ \\
\hline
\end{tabular}

En cuanto a la situación ocupacional se observa una realidad similar en los tres grupos de intensidad de pertenencia, donde más de un tercio trabaja en jornada completa y existe un nivel de desempleo cercano al 7\%, algo inferior al desempleo a nivel nacional. Una gran proporción se dedica a los quehaceres del hogar, lo que indicaría en términos generales que en la mayoría de los hogares hay un solo perceptor de ingresos. En complemento a ello, la encuesta CASEN 2003 identifica un 25\% de hogares indígenas con jefatura femenina.

\section{Resultados: Intensidad de pertenencia cultural e identidad mapuche}

De acuerdo a las hipótesis que guían este trabajo, relacionadas en primer término con la presencia de una dualidad en los mapuche entre la intensidad de la pertenencia a su cultura ancestral y la práctica o participación de sus ritos y costumbres; y en segundo lugar con la existencia de una ambivalencia cultural de los mapuche entre la pertenencia a una etnia y la identificación y anhelo de integración a una identidad nacional, se analizarán algunas preguntas de la encuesta que reflejan esta situación al interior de los grupos de distinta intensidad de pertenencia, así como en comparación con el grupo de control no mapuche.

\subsection{Intensidad de la pertenencia étnica: Adhesión simbólica o práctica de una cultura}

En primer lugar, al preguntar por la importancia de ciertos atributos para la mantención de la cultura mapuche y los rasgos más distintivos de una persona mapuche, transversalmente los tres grupos de intensidad de 
pertenencia atribuyen mayor relevancia a rasgos como el uso de la lengua, los apellidos, el conocimiento de la historia del pueblo mapuche o vivir en tierras mapuche. Aunque no siempre están presentes, se consideran elementos constituyentes de su cultura, que les dan un valor más bien simbólico y permanecen como parte del imaginario mapuche. No ocurre igual con otros atributos que tienen que ver directamente con la práctica de costumbres, pues la participación en ceremonias mapuche, los ritos matrimoniales, la vestimenta o ir donde la machi no son considerados de gran importancia para la conservación de la cultura.

Un hecho que constata esta dualidad entre la valoración simbólica de la cultura y la práctica de la misma tiene que ver con el uso de la lengua. Al preguntar en qué idioma se habla habitualmente con los niños pequeños en el lugar donde se vive, la mayoría contesta que en castellano, especialmente a medida que disminuye la intensidad de la pertenencia, como indica el Gráfico $N^{\circ} 2$.

Si bien lo anterior se relaciona con que son muy pocos los que hablan o al menos entienden el mapudungun, especialmente en los grupos de intensidad de pertenencia media y baja, llama la atención que más de la mitad de la muestra en los tres grupos de intensidad de pertenencia consideran que la enseñanza del mapudungun debiera ser obligatoria para los niños mapuche, como indica el Gráfico $\mathrm{N}^{\circ} 3$.

GRÁFICO No 2: ¿EN QUÉ IDIOMA HABLA HABITUALMENTE CON NIÑOS PEQUEÑOS EN EL LUGAR DONDE VIVE?

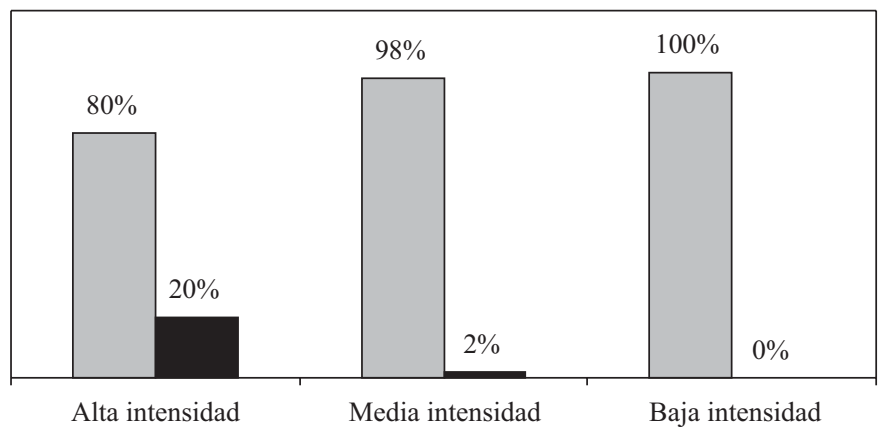

$\square$ Castellano $\square$ Lengua mapuche

Nota: Diferencias estadísticamente significativas con un 95\% de confianza en todos los casos. 
GRÁFICON ${ }^{\circ}$ 3: ¿LA ENSEÑANZA DE LA LENGUA MAPUCHE PARA NIÑOS MAPUCHE DEBIERA SER OBLIGATORIA O VOLUNTARIA?

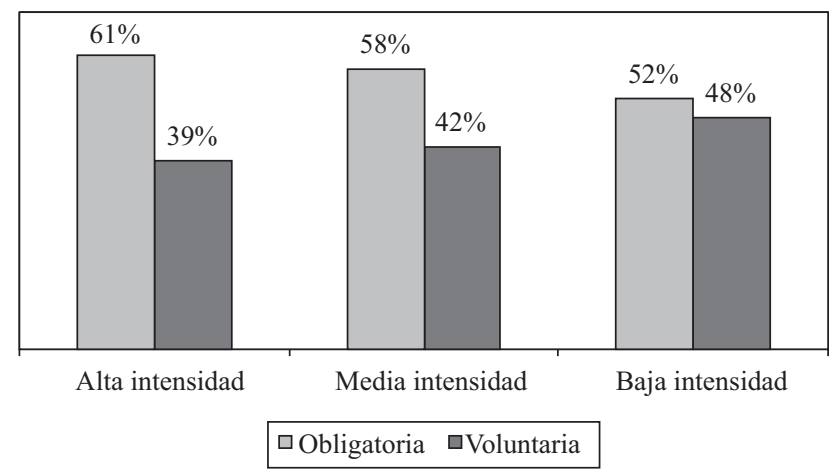

Nota: Diferencias estadísticamente significativas con un 95\% de confianza para la enseñanza obligatoria de la lengua mapuche (mapudungun) entre los grupos de alta y entre los de baja intensidad, y media y baja intensidad de pertenencia.

En este sentido, se puede afirmar que el sentimiento de pertenencia de los mapuche a su cultura va más allá de las prácticas mismas y que la pertenencia a la cultura mapuche coexiste con la pertenencia a una sociedad nacional mayor. $\mathrm{Al}$ respecto, muchos mapuche se definen a sí mismos como una mezcla entre mapuche y chileno, especialmente en el caso de los mapuche de baja intensidad de pertenencia, quienes alcanzan al 80\%, como se observa en el Gráfico $N^{0}$ 4. Sin embargo, entre los grupos de intensidad media y alta particularmente, un alto porcentaje se siente mapuche antes que chileno, lo cual muestra que en un conjunto que se autoconcibe como perteneciente a una cultura existen diferentes posturas a la hora de adherir a ella.

No ocurre lo mismo ante la pregunta por la procedencia de la pareja: en los tres grupos una alta proporción afirma tener pareja chilena o mezcla de mapuche y chileno, lo cual incide en la mayor apertura a convivir y dejarse permear por la cultura nacional.

En definitiva, los datos nos muestran que existe cierta apertura hacia la cultura dominante y una adaptación de los estilos de vida de los mapuche, pues casarse y formar familia con no mapuche y que los mapuche se vayan a vivir a la ciudad no son considerados como una amenaza para la conservación de la cultura. Al preguntar la opinión acerca de si estaría bien o mal que sus hijos o hermanos se casaran con personas no mapuche, no se 


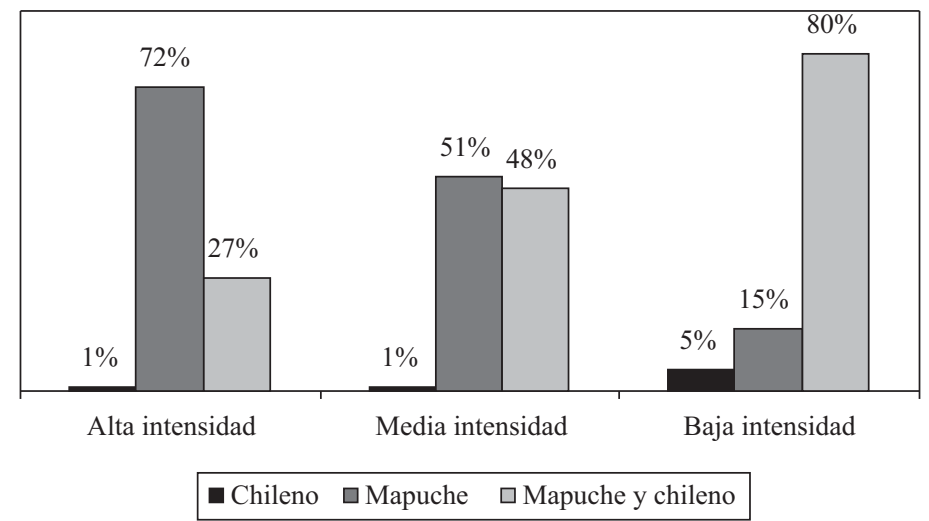

Nota: Diferencias estadísticamente significativas (95\% confianza) para todos los grupos que se sienten mapuche o una mezcla de mapuche y chileno. Para los que se sienten chilenos, solamente son estadísticamente significativas las diferencias entre los grupos de media y baja intensidad de pertenencia.

observa mayor resistencia, pues independientemente del grupo de pertenencia, a una alta proporción le parecería bien o le sería indiferente tal hecho, como lo muestra el Gráfico $\mathrm{N}^{0}$ 5, aunque esta proporción es algo menor para el grupo de alta intensidad de pertenencia.

Lo mismo ocurre para los mapuche rurales respecto a apoyar a un hijo si quisiera irse a vivir a la ciudad, pues como lo ilustra el Gráfico $N^{\circ} 6$, la mayoría lo apoyaría, aunque en el grupo de alta intensidad de pertenencia existe un 32\% que afirma que trataría de impedirlo. En este sentido, las expectativas de integración de los mapuche se traducen muchas veces en las oportunidades que puedan tener los hijos. Otro ejemplo de ello es que un alto porcentaje en todos los grupos afirma que le gustaría que sus hijos hicieran el servicio militar.

Hay otros rasgos que también son considerados de importancia a nivel declarativo para la mantención de la cultura, los cuales tienen que ver con ciertas demandas históricas del pueblo mapuche, como la reclamación de sus tierras, su reconocimiento constitucional o la no discriminación, todas ellas demandas que se han mantenido como una constante.

Sin embargo, en la práctica, estas demandas contrastan con ciertos valores más individualistas que sobresalen en temas como la propiedad de 
GRÁFICO N ${ }^{\circ}$ 5: ¿UD. CREE QUE ESTARÍA BIEN O MAL QUE SU HIJA O HERMANA DECIDIERA CASARSE CON UNA PERSONA NO MAPUCHE?

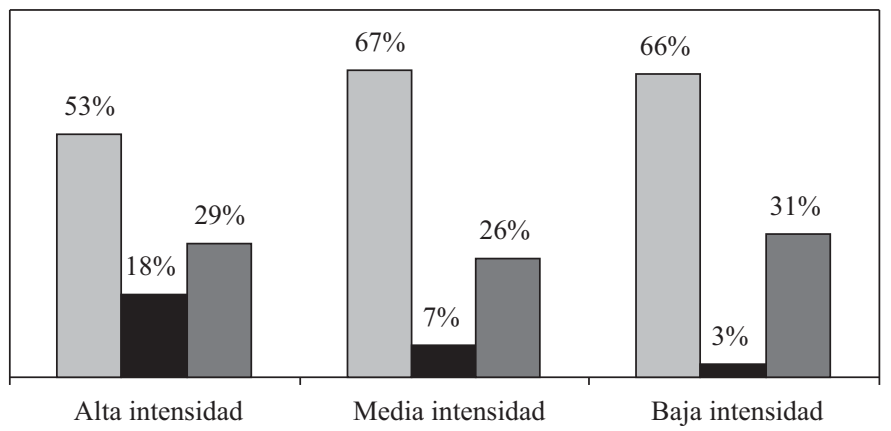

$\square$ Bien $\square$ Mal $\square$ Le da lo mismo

Nota: Diferencias estadísticamente significativas con el 95\% de confianza para la respuesta "bien" entre los grupos de alta y baja intensidad, y entre los grupos de alta y media intensidad de pertenencia.

GRÁFICO N ${ }^{\circ}$ 6: SI SU HIJO QUISIERA IRSE A VIVIR A UNA CIUDAD GRANDE EN BUSCA DE MEJORES OPORTUNIDADES, ¿USTED LO APOYA?*

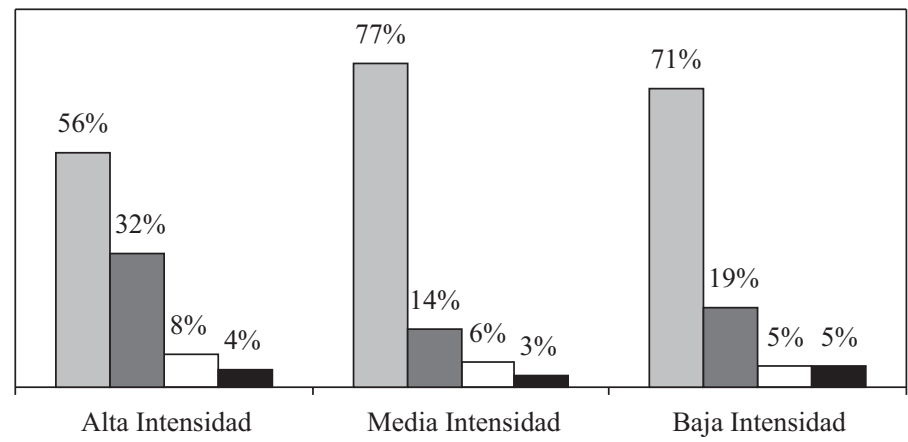

$\square$ Lo apoya $\square$ Trata de que no se vaya $\square$ No hace nada $\quad$ No sabe

*Esta pregunta sólo considera muestra de mapuche rurales.

Nota: Diferencias estadísticamente significativas con el 95\% de confianza para la respuesta "Lo apoya" entre los grupos de alta y baja intensidad, y entre los grupos de alta y media intensidad de pertenencia. 
la tierra, pues la mayoría de los mapuche encuestados, proporciones cercanas al $90 \%$ en los grupos de distinta intensidad de la pertenencia, preferiría que las tierras mapuche estuvieran en manos de las personas y sus familias en vez de la comunidad, aunque existe cierta preferencia a que las tierras pudieran venderse solamente entre mapuche.

Por otra parte, al consultar por los principales problemas de las personas mapuche a los que debería poner atención el gobierno, éstos son en gran parte demandas similares a las que conocemos de otros grupos sociales del país de similar condición socioeconómica. No obstante, el tema de la recuperación de las tierras indígenas sigue siendo prioritario para todos los grupos de intensidad de pertenencia, lo que responde nuevamente a esta pertenencia simbólica a un territorio ancestral presente en el imaginario mapuche, que hará que ésta se mantenga como una demanda permanente entre el pueblo mapuche y que coexista con otras demandas sociales relacionadas con la pobreza, la falta de educación, la discriminación, las pocas oportunidades de empleo y la falta de ayuda económica del gobierno. De este modo, pese a la persistencia de algunos temas de importancia histórica para los mapuche, sus necesidades sociales son igualmente prioritarias y son compartidas además con otros ciudadanos chilenos de grupos socioeconómicos semejantes.

\subsection{Ambivalencia cultural: Identidad étnica vs. identidad nacional}

Se ha afirmado que los mapuche, además de reconocerse como miembros de su pueblo original, también se reconocen como parte de una identidad mayor que podría identificarse como nacional. En este sentido los mapuche compartirían con el resto de los chilenos, o con distintos grupos de ellos, lugares de residencia, estilos de vida, escuelas donde asisten sus hijos, puestos de trabajo, entre otras cosas, lo que indica que no se diferencian mayormente de otros grupos del país y que presentan necesidades y expectativas similares.

Un ejemplo de ello se refleja al comparar los principales requerimientos sociales del grupo mapuche con los del grupo de control ante los esfuerzos del gobierno. En efecto, al preguntar por los principales problemas a nivel del país identificados por los mapuche, no se observan mayores diferencias con los señalados por el grupo no mapuche, ni con los problemas antes mencionados como propios de las personas de origen mapuche.

Como se observa en la Tabla $\mathrm{N}^{0} 5$, nuevamente los problemas identificados tienen relación en ambos casos con preocupaciones por la pobreza, por mayor empleo, educación y salud, y aparece la delincuencia como otra preocupación en la lista. De esta manera, se evidencia nuevamente que los 
TABLA N ${ }^{\circ}$ 5: $\quad$ PRINCIPALES PROBLEMAS A LOS QUE DEBIERA DEDICAR MAYOR ESFUERZO EL GOBIERNO

Muestra mapuche

Grupo de control

1. Empleo

1. Pobreza

2. Pobreza

2. Empleo

3. Educación

3. Delincuencia

4. Salud

4. Salud

5. Delincuencia

5. Educación

mapuche en tanto grupo social presentan demandas similares a las de otros ciudadanos chilenos en equivalente situación.

Otra comparación que resulta interesante con el grupo de control no mapuche tiene que ver con el nivel de confianza en las instituciones por parte de estos grupos. En un primer caso, como muestran las Tablas $\mathrm{N}^{\circ} 6 \mathrm{y}$ $\mathrm{N}^{\circ} 7$, aparece entre los mapuche una mayor confianza en las instituciones mapuche que en las instituciones nacionales, particularmente en lo que compete a autoridades comunitarias como el longko o la/el machi, con niveles de confianza de $58 \%$ y $57 \%$ respectivamente. Al respecto, estudios han demostrado que actualmente el respeto a estas autoridades se relaciona más bien con la condición simbólica que representan en tanto signos de la permanencia de elementos culturales mapuche, que con la efectividad real de estas instituciones en las decisiones sociopolíticas de las mismas ${ }^{5}$. Sin embargo, por otro lado se observan niveles similares de confianza para el caso de las municipalidades, lo cual indica que los municipios, dentro de las instituciones nacionales, tendrían para los mapuche una alta validación al igual que sus autoridades tradicionales, esta vez con alcances sociopolíticos reales.

También están dentro de las instituciones con mayores niveles de confianza en el grupo mapuche el Ad Mapu, que representa el conjunto de leyes y tradiciones de la cultura mapuche; la CONADI, institución gubernamental de desarrollo indígena, y el Consejo de Todas las Tierras, organización mapuche de mayor influencia nacional e internacional. Al comparar con la confianza que existe hacia instituciones nacionales, entre los primeros lugares después de los municipios se encuentran el gobierno, Carabineros, las iglesias y fuerzas armadas, y en las últimas posiciones el Congreso y los

${ }^{5}$ MINEDUC: “Identificación y descripción de Contenidos Culturales Aymara, Atacameños y Mapuche”, 2005. 
TABLA N ${ }^{\circ}$ 6: $\quad$ PORCENTAJE DE CONFIANZA EN INSTITUCIONES MAPUCHE*

Institución mapuche

Longko

Machi

Ad Mapu

CONADI

Consejo de Todas las Tierras

Identidad Territorial Lafkenche

Asociación Ñancucheo de Lumaco

Consejo Pu Werken Lof Budi

Coordinadora Arauco Malleco

Kallfülikan
Muestra mapuche

$58 \%$

$57 \%$

$55 \%$

$54 \%$

$54 \%$

$47 \%$

$43 \%$

$41 \%$

$41 \%$

$37 \%$

*Proporción que afirma tener mucha o bastante confianza en instituciones

TABLA N ${ }^{\circ} 7: \quad$ PORCENTAJE DE CONFIANZA EN INSTITUCIONES NO MAPUCHE*

Municipalidades

Gobierno

Carabineros

Iglesia evangélica

Fuerzas armadas

PYMES

Iglesia católica

Televisión

Sindicatos

Prensa

Grandes empresas

Ministerio público

Tribunales de justicia

Congreso

Partidos políticos

$\begin{array}{rr}58 \% & 59 \% \\ 51 \% & 49 \% \\ 49 \% & 54 \% \\ 48 \% & 44 \% \\ 47 \% & 51 \% \\ 44 \% & 49 \% \\ 43 \% & 47 \% \\ 32 \% & 34 \% \\ 31 \% & 30 \% \\ 28 \% & 30 \% \\ 27 \% & 28 \% \\ 24 \% & 25 \% \\ 20 \% & 29 \% \\ 18 \% & 17 \% \\ 9 \% & 8 \%\end{array}$

*Proporción que afirma tener mucha o bastante confianza en instituciones

partidos políticos, situación que se repite dentro del grupo de control, lo que indica la coherencia entre las percepciones de los mapuche y el resto de los chilenos.

En cuanto a las preferencias políticas de los grupos estudiados, se repite el desprestigio y desconfianza existente hacia los partidos políticos, pues una gran proporción tanto de mapuche como de no mapuche (sobre el 
$40 \%$ en ambos casos) afirma no identificarse con ninguna de las coaliciones políticas nacionales, no encontrándose además mayores diferencias entre los grupos en su adscripción política.

Por otra parte, la mayor confianza en las Iglesias católica y evangélicas se refleja en la fuerte participación de los mapuche en estas religiones. $\mathrm{Al}$ respecto, los datos nos indican que una alta proporción de ellos — sobre el 50\%- se dice perteneciente a la Iglesia católica. A las Iglesias evangélicas también pertenece un porcentaje importante, en torno al 35\%. En comparación con el grupo de control, se observa una mayor participación de los mapuche en Iglesias evangélicas con respecto a los no mapuche, no así en la Iglesia católica. Todo esto nos indica una vez más esta doble militancia entre una identidad étnica mapuche y el respeto por la conservación de una religiosidad indígena, y la pertenencia a una identidad nacional y la integración a sus valores.

Esta dualidad se observa también en temas de género. Como lo ilustra el Gráfico ํo 7, al preguntar la opinión personal respecto a la aceptación del aborto, si bien un 68\% lo considera como algo inaceptable, llama la atención la presencia de signos más liberales: un 30\% señala que dependería de las circunstancias, proporción similar al 37\% del grupo de control que afirma lo mismo. Sin embargo, en temas como la incorporación de la mujer al

GRÁFICO No 7: $\quad$ EN SU OPINIÓN PERSONAL, QUE UNA MUJER SE HAGA UN ABORTO, ¿ES ACEPTABLE, INACEPTABLE O DEPENDE DE LAS CIRCUNSTANCIAS?

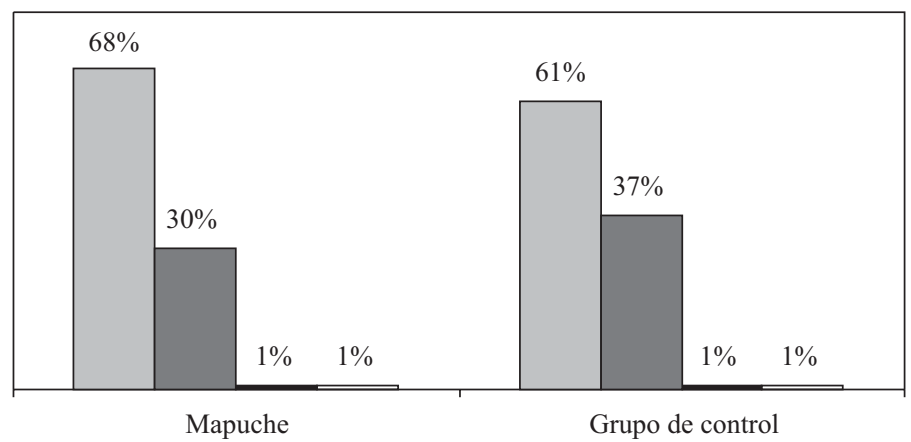

$\square$ Inaceptable $\square$ Depende $\square$ Aceptable $\square$ No sabe

Nota: Diferencias estadísticamente significativas con el 95\% de confianza entre los grupos mapuche y no mapuche para las respuestas "inaceptable” y "depende de la circunstancia”. 
mundo laboral existe entre los mapuche una tendencia más conservadora que lleva a pensar que el trabajo de la mujer resiente la vida familiar y de la pareja, valorándose el trabajo doméstico de la mujer por sobre su independencia económica.

En este sentido, se percibe en las distintas declaraciones y posturas de los mapuche un sentimiento de integración a una identidad común a otros grupos sociales, en coexistencia con la pertenencia y la conservación de su cultura. De hecho, al consultar a los mapuche qué tan integrados se sienten a Chile, un alto porcentaje dice sentirse plenamente integrado, aunque en menor medida que el grupo de control no mapuche, como muestra el Gráfico $\mathrm{N}^{\circ} 8$.

En esta misma línea se observan apreciaciones similares al preguntarles a los mapuche su opinión respecto a la autonomía o a la integración de las comunidades mapuche al resto de Chile, donde cerca de un $80 \%$ afirma que las comunidades debieran integrarse más. De esta manera, se refuerza la idea de que existe en el imaginario mapuche un anhelo de integración a la sociedad mayor, aun cuando se reconozca la pertenencia a una cultura en particular y se mantenga con fuerza la identidad étnica que los constituye.

GRÁFICO No 8: ¿ ¿DIRÍA USTED QUE SE SIENTE PLENAMENTE INTEGRADO, PARCIALMENTE INTEGRADO O NADA INTEGRADO A CHILE?

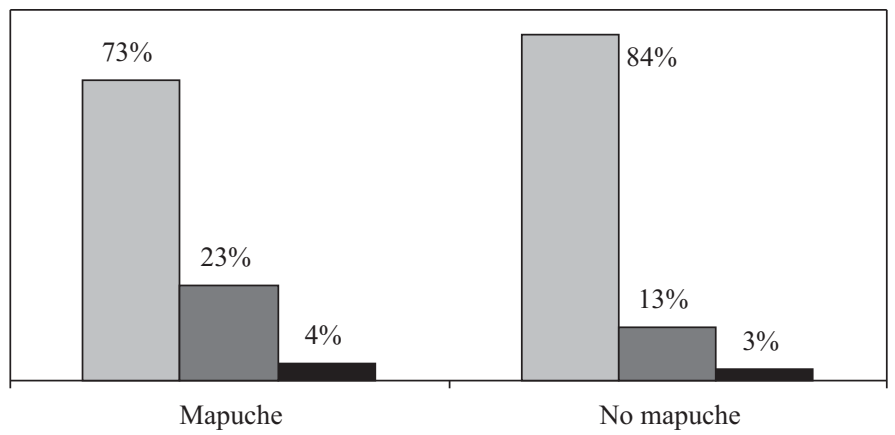

$\square$ Plenamente integrado $\square$ Parcialmente integrado $\square$ Nada integrado

Nota: Diferencias estadísticamente significativas con el 95\% de confianza entre los grupos mapuche y no mapuche para las respuestas "plenamente integrado" y "parcialmente integrado". 


\section{Conclusiones}

Al estudiar a los mapuche de acuerdo a las intensidades de pertenencia a su cultura, se puede advertir que entre ellos existen grupos que viven y sienten su cultura de manera diferente. Sin embargo, lo interesante de constatar es que, aunque algunos mapuche vivan con mayor intensidad su cultura que otros, todos quienes se autodeclaran pertenecientes a la etnia adhieren referencialmente a una misma cultura originaria, valorando enormemente la conservación de ciertos rasgos, tales como: la tierras ancestrales, aunque no vivan físicamente en ellas; la lengua mapuche, aunque no la hablen ni la entiendan; el linaje, aunque hayan formado familia con no mapuche; la adherencia a una cosmovisión y a una historia común, aunque no participen de los ritos ni ceremonias mapuche. En este sentido, si bien existe una estimación simbólica de ciertos rasgos culturales, aun cuando se viva en la ciudad o exista un aparente proceso de aculturización, se percibe una fuerte persistencia de estos elementos ancestrales en el imaginario mapuche, que apelan de una u otra manera a la conservación de una cultura por parte de sus integrantes.

No obstante, esta valoración y pertenencia a la cultura mapuche coexiste con un anhelo permanente de integrarse a una cultura mayor, lo cual se refleja en que las percepciones que tienen las personas mapuche de la realidad nacional y de sus propias necesidades no se diferencian mayormente de las de otros grupos sociales que viven en condiciones similares en nuestro país. Tal como se ha discutido a lo largo de este trabajo, los mapuche comparten una situación socioeconómica con otros grupos de bajos estratos sociales, que presentan escasos niveles de educación y calificación y que viven en condiciones precarias de habitabilidad, especialmente en zonas rurales. En este marco, sus demandas sociales coinciden y se relacionan principalmente con temas como la pobreza, el empleo, la salud y la educación; aunque permanecen entre los mapuche reclamaciones históricas por sus tierras ancestrales o algunos derechos políticos.

Por otra parte, la añoranza cultural de un pasado ancestral se relaciona más bien con apreciaciones y percepciones a nivel declarativo que con prácticas específicas, pues una gran proporción de mapuche no cultivan mayormente su cultura. Sin embargo, si bien las preguntas de la encuesta apuntan más hacia percepciones que a conductas, podemos distinguir entre las respuestas algunas opiniones que, al estar mediadas por la experiencia misma de los mapuche, nos estarían indicando que en algunas ocasiones la balanza se inclina más hacia la integración a la sociedad mayor, sin obviar la pertenencia a su cultura. Ejemplos de ello se dejan ver en la apertura de los 
mapuche a formar familia con personas no mapuche, en las expectativas de integración de sus hijos, en la creencia en la propiedad individual antes que en la propiedad comunitaria o en los mayores deseos de integración de las comunidades mapuche al país, todas opiniones que pueden estar influenciadas en gran parte por las mismas vivencias cotidianas de los encuestados y que demuestran la capacidad de una cultura de adaptarse y de incorporar nuevas formas de vida a su pertenencia cultural. En este sentido, se distingue una doble identidad que convive entre los mapuche, aquella que valora y realza la pertenencia a una cultura ancestral, y otra que se adapta y hace suyos elementos de una cultura mayor que forma parte de una identidad nacional.

En definitiva, el presente análisis devela entre los mapuche una dicotomía entre la pertenencia a su cultura y el anhelo de integración a la sociedad nacional. Esta dicotomía no es extraña a la idiosincrasia de la sociedad chilena, pues al revisar resultados de otros estudios, como el "Informe de Desarrollo Humano" del PNUD del año 2002, se señala que en el imaginario colectivo de los chilenos los pueblos indígenas aparecen como raíz de nuestra nacionalidad y cultura, y que existe una actitud positiva hacia sus reivindicaciones. Sin embargo, al mismo tiempo los chilenos consideran que el mayor desarrollo de Chile en comparación con otros países de la región se debe a la baja importancia relativa de la población indígena del país. Por otra parte, la reciente encuesta bicentenario UC-Adimark 2006 muestra que un $67 \%$ de los chilenos cree que es mejor que los mapuche mantengan su cultura, en contraposición a que se adapten. De esta manera se advierte que la temática mapuche en Chile también presenta esta ambivalencia entre la pertenencia a una cultura indígena y el deseo de integración al resto país.

En este sentido, análisis como el presente demuestran que entre los mapuche el deseo de conservación de su cultura convive con un simultáneo anhelo de integración a la identidad nacional, y hay un grupo mayoritario al interior del pueblo mapuche que, aún aspirando a una mayor integración, se siente igualmente parte de una etnia que añora su conservación como patrimonio cultural de nuestro país. Para una mayor profundidad en el estudio de estos temas habría que analizar a través de estudios etnográficos lo que ocurre al interior de estos grupos, considerando también la complejidad y diversidad que existe además dentro del mundo mapuche. Por el momento, el análisis de una encuesta nacional mapuche permite aproximarse de manera general a una realidad existente, ayudando a comprender a grandes rasgos los diferentes grupos e intensidades con que los mapuche viven su cultura en nuestro país. 
A partir de las reflexiones anteriores y en un terreno más concreto, es conveniente hacer algunos alcances útiles para el campo de las políticas públicas, destacando al menos tres puntos relevantes de considerar a la hora de pensar en el rol del Estado en materia indígena.

- $\quad$ Como hemos analizado en este estudio, los mapuche, independientemente de la intensidad con que vivan su cultura, se sienten por igual pertenecientes a un pueblo ancestral, del cual quieren ser reconocidos como originarios. Elementos culturales referenciales como la tierra, la lengua o la sangre son fundamentales en estos términos y constituyen parte importante de la demanda mapuche por ser reconocidos como tales. En este sentido, un desafío para las políticas indígenas y la sociedad chilena en general es buscar métodos adecuados de reconocimiento cultural del pueblo mapuche, entendimiento de sus necesidades y expectativas, a partir de las cuales se puedan diseñar programas para la preservación y prolongación de su cultura.

- $\quad$ En segundo lugar, se ha constatado ya la coexistencia en el imaginario mapuche del deseo de conservación de su cultura junto con un anhelo de integración a la sociedad mayor. En este marco, las políticas indígenas deberían considerar un enfoque intercultural, en el sentido de la mantención de las culturas originarias en el diálogo e interacción permanente con la cultura nacional. Es decir, políticas que favorezcan un proceso de convivencia entre las culturas y que lleven al constante enriquecimiento mutuo y legitimación de los saberes de cada una. En este sentido, en el ámbito de la educación son fundamentales planes públicos que potencien la educación intercultural bilingüe. También debe considerarse la interculturalidad en el plano de la salud y en el ámbito productivo, en donde la interacción y el intercambio de elementos culturales es fundamental para una mejor calidad de vida de la población indígena, junto con la protección de sus culturas.

- $\quad$ Por último, cabe señalar que dada la complejidad propia del mundo mapuche, existen distintos grupos en su interior, los cuales viven y sienten con distintas intensidades la pertenencia a su cultura. Para efectos de intervención pública, deben tomarse en cuenta estas diferencias y saber reconocer las verdaderas demandas de estos grupos. Muchas veces se ha asociado el mundo indígena al ámbito rural, cuyos miembros si bien presentan altos niveles de intensidad de pertenencia a su cultura, también pertenecen a una sociedad mayor, 
con la cual comparten valores y prácticas. En este sentido, las políticas públicas deben desmitificar a la sociedad indígena rural y conocer en mayor profundidad sus necesidades y expectativas, las que, como hemos visto, no se diferencian mayormente de las de otros grupos indígenas urbanos, o de otros grupos de similares condiciones socioeconómicas en nuestro país.

\section{REFERENCIAS BIBLIOGRÁFICAS}

Cañulef, Eliseo: Introducción a la Educación Intercultural Bilingüe en Chile. Temuco: Instituto de Estudios Indígenas Universidad de La Frontera, 1998.

Centro de Estudios Públicos (CEP): “Estudio de Opinión Pública: Los Mapuche Rurales y Urbanos Hoy. Mayo 2006”. Santiago: CEP, 2006.

MIDEPLAN (Ministerio de Planificación Nacional): Encuesta de Caracterización Socioeconómica Nacional y Población Indígena (CASEN). Documento preparado por la Coordinación de Políticas y Programas Indígena de la Subsecretaría del Ministerio de Planificación. Noviembre 2005. En www.mideplan.cl

MINEDUC (Ministerio de Educación): Identificación y Descripción de Contenidos Culturales Aymara, Atacameños y Mapuche. Santiago, 2005.

PNUD: "Informe de Desarrollo Humano en Chile". En Nosotros los Chilenos: Un Desafío Cultural. Santiago: Editorial PNUD, 2002.

UC-Adimark: “Encuesta Bicentenario 2006”. En www.uc.cl

Universidad de Chile, Facultad de Ciencias Económicas y Administrativas, Departamento de Economía: "Línea Base en Comunidades Indígenas Programa Orígenes”, 2004.

Zúñiga, Fernando: Mapudungun. El Habla Mapuche. Santiago: Centro de Estudios Públicos, 2006. 